\title{
Modelo instruccional Blended- Flipped: personalización, flexibilización y metacognición para la nivelación en inglés en la educación superior
}

Primer semestre de 2021 - pp. 107-122

Segunda época N.

\author{
Blended-Flipped \\ Instructional Model: \\ Personalization, \\ Flexibility and \\ Metacognition \\ for the Leveling of \\ English in Higher \\ Education
}

\author{
Modelo \\ instrucional \\ Blended-Flipped: \\ personalização, \\ flexibilidade e \\ metacognição para \\ 0 nivelamento do \\ inglês no ensino \\ superior
}

María Catalina Caro Torres* http://orcid.org/0000-0002-7575-1111

Diana Angélica Parra Pérez** https://orcid.org/0000-0002-3068-0970

Alfredo José Averanga Murillo*** https://orcid.org/0000-0002-1145-4433

Nelson Javier Corredor Plazas*** https://orcid.org/0000-0001-9002-9525

Rosa Alejandra Medina Riveros***** https://orcid.org/0000-0001-9601-5319

* $\quad$ Magíster en Didáctica del Inglés con Énfasis en Ambientes de Aprendizaje Autónomo. Jefe de Área de Apoyo al Aprendizaje de Inglés, Departamento de Lenguas y Culturas Extranjeras, Universidad de La Sabana.

Correo electrónico: maria.caro1@unisabana.edu.co

** Magíster en Didáctica del Inglés con Énfasis en Ambientes de Aprendizaje Autónomo. Directora de Studium, Centro de Recursos e Investigación en Idiomas del Departamento de Lenguas y Culturas Extranjeras de La Universidad de La Sabana.Universidad de La Sabana. Correo electrónico: diana.parra5@unisabana.edu.co

*** Magíster en Informática Educativa. Gestor de recursos de digitales en el Centro de Recursos e Investigación en Idiomas StudiumUniversidad de La Sabana.

Correo electrónico: alfredo.averanga@unisabana.edu.co

*** Licenciado en Informática educativa. coordinador de recursos digitales en Studium, Centro de Recursos e Investigación en Idiomas, Universidad de La Sabana.

Correo electrónico: nelson.corredor@unisabana.edu.co

***** Doctora en Educación. Universidad de La Sabana.

Correo electrónico: rosa.medina@unisabana.edu.co

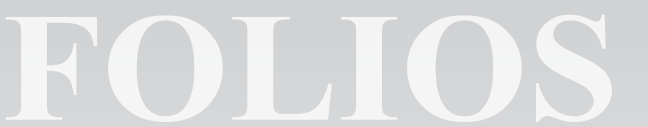

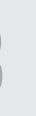




\title{
Resumen
}

Este artículo de investigación presenta una exploración de un modelo instruccional híbrido y de aula invertida para el aprendizaje de inglés como lengua extranjera y sus alcances en la atención de las necesidades de nivelación de un grupo de estudiantes universitarios. El modelo nace como una estrategia de éxito académico para estudiantes universitarios que requieren estudiar inglés con una opción ajustable tanto a sus procesos de aprendizaje como a sus circunstancias de vida. Con un enfoque de corte cualitativo, se recolectaron los datos y se siguieron los postulados propuestos en la teoría fundamentada para el análisis constante y la codificación de los datos. Los resultados de investigación indican que los estudiantes perciben que el modelo instruccional híbrido-invertido les permitió superar barreras afectivas y de tiempo, mediante la flexibilidad y la personalización, además de incentivar comportamientos metacognitivos. Lo anterior permitió a los investigadores concluir que el modelo propuesto favorece el aprendizaje de inglés y que su enfoque híbrido-invertido demuestra que la tecnología es un medio facilitador en el proceso de flexibilización y adaptación de las prácticas de enseñanza a las necesidades de los aprendices de lenguas.

\author{
Palabras clave \\ educación superior; aprendizaje híbrido; enseñanza asistida por \\ ordenador: enseñanza de idiomas; aula invertida
}

\begin{abstract}
This research article presents an exploration of the scope of an instructional model of English as a foreign language created in a hybrid environment and with a flipped classroom approach, in addressing the leveling needs of a group of university students. The model was born as an academic success strategy for university students who need to study English with an adjustable option both to their learning processes and to their life circumstances. Data collection followed a qualitative approach; and the analysis involved a constant analysis and coding of the data implementing the postulates of grounded theory. The research results indicate that students perceive that the hybrid-flipped instructional model allowed them to overcome time and affective barriers through flexibility and personalization. The model also encouraged students' metacognitive behaviors. These findings allowed the researchers to conclude that the proposed model favors English learning and that the hybrid-flipped approach demonstrates that technology facilitates the process of flexibilization and adaptation of teaching practices to the language learners' needs.

\section{Keywords}

higher education; blended learning; computer assisted language learning; language teaching; flipped classroom
\end{abstract}

\section{Resumo}

Este artigo de pesquisa apresenta uma análise exploratória sobre a abrangência de um modelo instrucional do inglês como língua estrangeira, criado em um ambiente híbrido e com uma abordagem de aula invertida, para atender às necessidades de nivelamento de um grupo de estudantes universitários. 0 modelo nasceu como uma estratégia de sucesso acadêmico para estudantes universitários que precisam estudar inglês com uma opção ajustável tanto ao processo de aprendizagem quanto às circunstâncias da vida. Mediante uma abordagem qualitativa, foram coletados os dados e se seguiram os postulados propostos na teoria fundamentada, com base na constante análise e codificação dos dados. Os resultados da pesquisa indicam que os alunos percebem que o modelo instrucional híbrido-invertido lhes permitiu superar barreiras afetivas e de tempo por meio de flexibilidade e personalização, além de incentivar comportamentos metacognitivos. 0 acima referido permitiu que os pesquisadores concluíram que o modelo proposto favorece o aprendizado do inglês e que sua abordagem híbrida-invertida demonstra que a tecnologia é um meio facilitador no processo de flexibilização e adaptação das práticas de ensino às necessidades dos alunos línguas.

\section{Palavras chave}

ensino superior; aprendizagem híbrida; ensino assistido por com-

putador; ensino de idiomas; sala de aula invertida 


\section{Introducción}

La tecnología y la educación podrían definirse como dos dinámicas coexistentes en un proceso de evolución continua en la que la educación, desde su interés por adaptarse a las necesidades del nuevo mundo, y la tecnología, desde su rol facilitador de procesos académicos, se entrelaza de manera flexible en muchos contextos de enseñanza-aprendizaje. El aprendizaje del inglés como lengua extranjera es uno de dichos contextos en el que la integración de la tecnología ha llegado a permear.

El modelo instruccional propuesto en este estudio nace en respuesta a la identificación de las necesidades y circunstancias determinantes sobre el éxito académico en la asignatura de inglés de estudiantes universitarios. Los antecedentes indican pérdidas reiteradas de los cursos de inglés e inasistencia alta a clase por inicio de sus prácticas laborales. Para ellos, a partir del 2007 y hasta la actualidad, se fundamenta la alternativa de estudio "Programa de Trabajo Independiente: Plan Umbrella", un programa en el que los niveles de inglés se cursan de manera autónoma a través de recursos de auto-acceso y con el acompañamiento de un tutor.

El modelo integra la tecnología mediante el uso de un ambiente virtual en la plataforma Moodle, que al ser combinado con el ambiente presencial, permite la construcción de un programa híbrido con un enfoque de aula invertida. Este artículo presenta el proceso de análisis, diseño, desarrollo e implementación del modelo instruccional de Plan Umbrella para la enseñanza-aprendizaje de inglés como lengua extranjera en un contexto universitario. Además, indaga sobre los alcances del modelo al resolver la pregunta de investigación: ¿Qué percepción tienen los estudiantes sobre una propuesta de modelo instruccional para un ambiente híbrido de aprendizaje de inglés como lengua extranjera con un enfoque de aula invertida?

En el siguiente apartado se presentarán los fundamentos teóricos que explican los alcances de integrar el aprendizaje híbrido en el aula de inglés, además de algunos resultados de su implementación en otros estudios. Luego, se expondrá sobre la importancia de flexibilizar los procesos de enseñan-
Modelo instruccional Blended-Flipped: personalización, flexibilización y metacognición para la nivelación en inglés en la educación superior María Catalina Caro Torres / Diana Angélica Parra Pérez / Alfredo José Averanga Murillo / Nelson Javier Corredor Plazas / Rosa Alejandra Medina Riveros

za-aprendizaje y su conexión con la integración de las nuevas tecnologías.

\section{Marco teórico}

\section{Aprendizaje híbrido}

Los ambientes de aprendizaje híbridos contrarrestan muchas de las falencias de los sistemas de educación tradicionales, puesto que proveen estructuras de redes flexibles y son considerados como un modelo favorable para el aprendizaje, especialmente para la educación superior (Alonso, López, Manrique y Viñes, 2005; Pavla, Hana y Jan, 2015). Las investigaciones en este campo demuestran que el aprendizaje híbrido beneficia diferentes escenarios de aprendizaje, entre estos, los cursos ofrecidos a estudiantes que inician el periodo de adaptación a la vida universitaria, o que se preparan para su periodo de prácticas laborales (Sriarunrasmee, Techataweewan y Mebusaya, 2015).

En el mundo digital en el que vivimos el aprendizaje híbrido se convierte en un modelo prometedor para el aprendizaje de lenguas extranjeras, ya que propende por el desarrollo de habilidades comunicativas (Janthon, Songkran y Koraneekij, 2015; Yalçınkaya, 2015). Por ejemplo, en la implementación de dos cursos de inglés en modalidad híbrida, Regalón y Leyva (2014) reportan un impacto pedagógico positivo evidenciado en el mejoramiento de la calidad de las clases y en la obtención de resultados más altos en las notas de los estudiantes.

Otros estudios en este campo también reportan el cambio favorable del rol del profesor en los escenarios de enseñanza híbridos, pasando de ser un conferencista y expositor a un guía, un tutor, o un mentor (Klimova y Kacetl, 2015; Krasnova y Demeshko, 2015), y en algunos casos, diseñador (Porter y Graham, 2016). El nuevo rol involucra habilidades del docente para motivar el aprendizaje (Avello y Duart, 2016; Kofar, 2016) e incentivar el desarrollo de habilidades de auto-conocimiento, auto-dirección, auto-regulación (Broadbent, 2017; Zhu, Au y Yates, 2016), e incluso de auto-perfección (Krasnova y Demeshko, 2015). 
En el ámbito de la educación superior, los estudios arrojan luces sobre los elementos que caracterizan a los ambientes híbridos de aprendizaje. Osorio (2010) encontró que los cursos desarrollados en un ambiente híbrido se caracterizaron por ser constructivistas y orientados, en su mayoría, al desarrollo de actividades colaborativas, comunicativas y de trabajo en grupo, lo cual coincide con lo encontrado por Matukhin y Zhitkova (2015) en un programa de maestría. Otro hallazgo significativo se relaciona con la percepción positiva de los estudiantes frente a los cursos híbridos, quienes los asocian con la construcción del conocimiento, el desarrollo de tareas auténticas en contextos significativos (reales), la variedad en la interacción (Osorio, 2010), y la oportunidad de desarrollar la autonomía, al fomentar la responsabilidad con su propio aprendizaje (Hubackova y Semradova, 2016).

Los desempeños de los estudiantes que participan en los procesos de aprendizaje en ambientes híbridos comparados con los de ambientes completamente virtuales han demostrado ser mejores (Thai, De Wever y Valcke, 2017). Se resalta del modelo híbrido, la posibilidad de crear escenarios de aprendizaje centrados en el estudiante, que propenden por el desarrollo de habilidades cognitivas (Khlaisang y Likhitdamrongkiat, 2015), y que contribuyen a la humanización, la personalización y la diferenciación de los procesos educativos (Matukhin y Zhitkova, 2015).

\section{Aula invertida}

El aula invertida se ha adoptado principalmente en modelos de aprendizaje híbrido y en contextos de educación superior, con el fin de cambiar las experiencias de aprendizaje (Sohrabi y Iraj, 2016), mejorar los desempeños y las técnicas de resolución de problemas, promover la participación y la interacción (Vidal, Rivera, Nolla, Morales y Vialart, 2016), y desarrollar habilidades tanto colaborativas como de autodirección (Blau y Shamir-Inbal, 2017; El-Banna, Whitlow y McNelis, 2017; Gilboy, Heinerichs y Pazzaglia, 2015).

Entre las fortalezas del aula invertida se encuentran el incremento de la motivación, el interés y la autonomía de los estudiantes (Gilboy, Heinerichs y
Pazzaglia, 2015; Sun, Xie y Anderman, 2018; Yang, Yin y Wang, 2018). En el aprendizaje de lenguas extranjeras se encontraron beneficios en las competencias comunicativa, sociocultural e interaccional (Jeong, 2017). También se informan mayores oportunidades para desarrollar habilidades de alto orden mediante la participación, la construcción de conocimiento, la interacción y las oportunidades de proveer y recibir retroalimentación (Blau y Shamir-Inbal, 2017).

El aula invertida ha demostrado tener efectos positivos sobre los desempeños de los estudiantes. Sergis, Sampson y Pelliccione (2018) señalan que este enfoque es efectivo para incrementar los desempeños de estudiantes que han presentado dificultades al tomar cursos completamente presenciales. Los aprendices pueden adaptar sus procesos de estudio al tiempo y ritmo de aprehensión individuales, y esto incide positivamente en su preparación para las clases presenciales (Gilboy, Heinerichs y Pazzaglia, 2015).

La inclusión gradual de contenidos y actividades en el aula invertida también ayudaría a los estudiantes a adaptarse y a tener mayor éxito (Hao y Lee, 2016). La efectividad del aula reside en cierta medida en un diseño instruccional que apunte a las necesidades reales y al perfil de aprendizaje de los estudiantes (Kakosimos, 2015) para facilitar el aprendizaje (Evseeva y Solozhenko, 2015) y fomentar la participación (Thai, De Wever y Valcke, 2017). Por lo tanto, en el aula invertida el docente también cumple un rol importante como diseñador (GarcíaSánchez y Santos-Espino, 2017; Porter y Graham, 2016; Wang, 2017).

Uno de los retos que enfrentan los estudiantes en el aula invertida es la ausencia del docente para aclarar dudas o instrucciones durante el desarrollo de las actividades previas a la clase (Gilboy, Heinerichs y Pazzaglia, 2015); no obstante, desde el aspecto pedagógico, esto es una oportunidad para promover la autonomía del estudiante (Chilingaryan y Zvereva, 2017; El-Banna, Whitlow y McNelis, 2017; Jeong, 2017). Wang, (2017), en concordancia con Vidal et al. (2016), plantean el uso de la auto-reflexión y la autoevaluación como mecanismos 
para que el rol de aprendiz pasivo cambie a uno completamente activo.

\section{Flexibilización del aprendizaje}

La flexibilización de los procesos de enseñanza-aprendizaje en la educación superior ha sido un foco de investigación permanente en los últimos años, sustentado en la necesidad de apoyar a los estudiantes en la búsqueda de formas propias de flexibilización que los preparen para enfrentar este mundo "inestable" (Barnett, 2014). La flexibilidad requiere de instituciones abiertas a operacionalizar un método altamente receptivo y personalizado, que se interese en conocer las necesidades de los aprendices (Butcher y Rose-Adams, 2015).

También se relaciona con los avances y usos de la tecnología en el ámbito educativo, puesto que la tecnología en sí misma facilita el aprendizaje flexible y provee oportunidades de aprendizaje personalizadas (Papadima-Sophocleous y Loizides, 2016). Se ha encontrado que los ambientes virtuales de aprendizaje incluyen mayor flexibilización que los ambientes tradicionales de aprendizaje (Ammar, 2017; Parra y Medina, 2014). Existen grupos de estudiantes que manifiestan su preferencia por las clases en línea resaltando su flexibilidad en cuanto al tiempo y lugar de estudio (Wright, 2017). Los MOoc (Massive Open Online Courses) permiten una flexibilidad considerable para la organización del aprendizaje (Liu, 2016; Loya et al., 2015). En el ámbito del diseño instruccional para ambientes híbridos se resalta la flexibilidad como un elemento clave en el éxito en el aprendizaje de lenguas extranjeras (Hirata y Hirata, 2008) y como una razón importante para promover dichos ambientes de aprendizaje (Kofar, 2016).

Los estudios sobre la conceptualización de la flexibilidad y la participación de los docentes de inglés en ambientes de aprendizaje de lenguas concluyen que promover estrategias de manejo y construcción de conocimiento es clave para el desarrollo de la creatividad y la participación de los docentes de lengua en procesos de aprendizaje integrales, reflexivos y de alto orden (Ammar, 2017; Keong y Subhi, 2015).

\section{Metodología}

\section{Construcción del modelo híbrido y de aula invertida}

El modelo instruccional híbrido-invertido presentado aquí adoptó para su desarrollo los parámetros del sistema de diseño instruccional ADDIE creado por el Centro de Tecnología Educativa en la Universidad Estatal de Florida en 1975 (Clark, 2014). Su denominación indica las cinco fases de las que se compone: Análisis, Diseño, Desarrollo, Implementación y Evaluación. Las primeras cuatro fases se desarrollan de forma progresiva, mientras que la última fase (Evaluación) tiene presencia dinámica en todo el desarrollo, logrando así un modelo de instrucción de carácter flexible (Clark, 2014).

\section{Fase de análisis}

El análisis de necesidades busca identificar el problema de instrucción, caracterizar a la audiencia en sus conocimientos, habilidades, propósitos y restricciones de aprendizaje, así como establecer las consideraciones pedagógicas; además, se verifican las posibles opciones de presentación o entrega de la instrucción para definir el ambiente de aprendizaje. En el análisis de necesidades que dio origen al modelo híbrido-invertido, encontramos que algunos estudiantes no estaban alcanzando las competencias requeridas en inglés, evidenciado en pérdidas reiteradas de uno o más cursos de inglés, inasistencias a clase o cancelación de los cursos por cruce de los horarios de clase con sus prácticas laborales o con otras asignaturas de su carrera. En consecuencia, se optó por un modelo de instrucción enfocado en dos frentes: 1) la flexibilización del programa de inglés ya existente, para disminuir las inasistencias o cancelación de la asignatura, y 2) la personalización del aprendizaje, para nivelar a los estudiantes en las competencias que no estaban logrando en el programa presencial de inglés.

\section{Fase de diseño}

En esta fase se desarrolla sistemáticamente un proceso de aprendizaje que responda a las necesidades identificadas en la fase de análisis. El producto de 
esta fase es un modelo del proceso de aprendizaje que contiene, entre otros elementos: los objetivos de aprendizaje, los desempeños a evaluar y la secuencia de aprendizaje. Para esta fase utilizamos las competencias establecidas en el programa presencial de inglés de la institución, y que se encuentran alineadas a los niveles A1.1 hasta B2.1 del Marco Común
Europeo de Referencia para las lenguas - MCER (Consejo de Europa, 2001). Seleccionamos y organizamos los contenidos de manera modular para ajustarlos a un ambiente híbrido compuesto por un $78 \%$ de la instrucción en el eLearning Management System (LMS) Moodle, y un 22 \% de la instrucción en el Campus Universitario (ver figura 1).

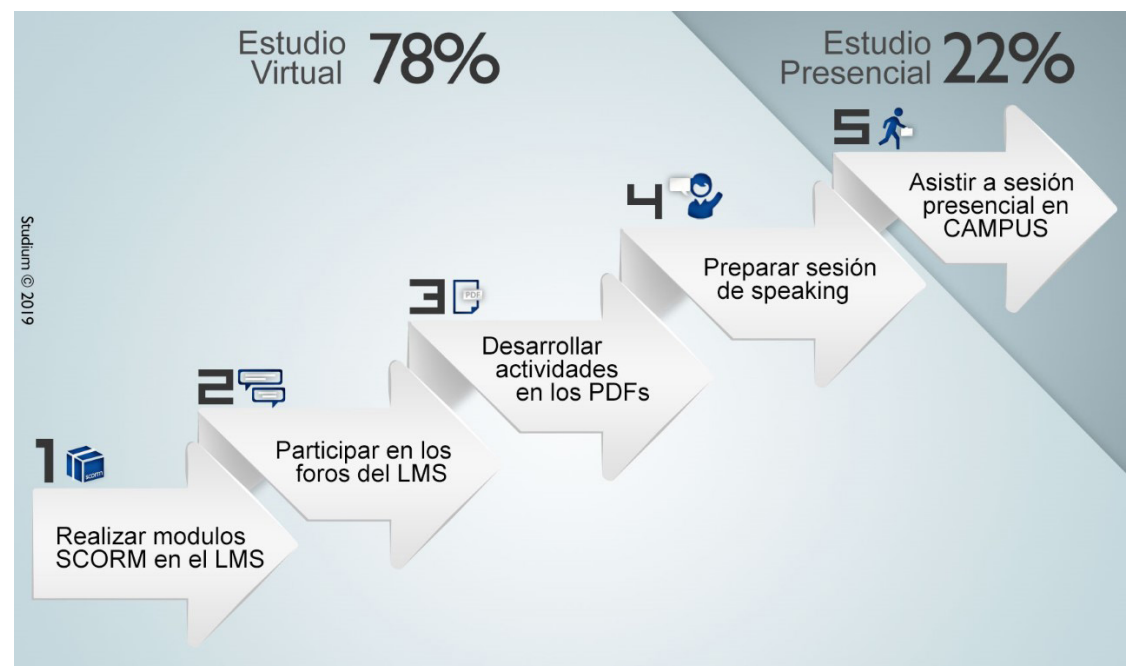

Figura 1. La secuencia de aprendizaje con un enfoque híbrido-invertido.

Fuente: elaboración propia.

Para estandarizar el diseño y guiar a los docentes en la secuencia didáctica de los contenidos, se crearon formatos de guiones con una secuencia de aprendizaje enmarcada en los nueve eventos de diseño instruccional propuestos por Gagné y Dick (1983): 1) captar la atención, 2) informar el objetivo, 3) activar los aprendizajes previos, 4) presentar el contenido, 5) guiar el aprendizaje, 6) provocar los desempeños, 7) proveer realimentación, 8) evaluar los desempeños y 9) mejorar la retención y la transferencia.

\section{Fase de desarrollo}

En esta fase se crea el material instruccional teniendo en cuenta las secuencias didácticas estructuradas en las dos fases anteriores, con el ánimo de alcanzar así el desarrollo de un entorno de aprendizaje completo. El resultado final es la plataforma de aprendizaje: el contenido, las lecciones, las ayudas, y los medios o recursos de apoyo que favorecen el aprendizaje y rendimiento de los estudiantes. $\mathrm{Al}$ seguir los nueve eventos para el diseño instruccional (Gagné y Dick, 1983) se desarrollaron 12 módulos de aprendizaje por cada uno de los siete niveles del programa. Cada módulo se estructura con: a) un paquete de actividades de auto-acceso en formato Sharable Content Object Reference Model (sCORM), b) una hoja de trabajo (Worksheet) en formato Portable Document Format (PDF), c) un foro de interacción, d) una actividad de preparación para la interacción oral en clase y e) una clase presencial.

Las actividades del SCORM apuntan a los primeros cinco eventos del modelo de diseño instruccional de Gagné y Dick (1983). La hoja de trabajo (Worksheet) y el Foro abordan los eventos seis y siete, ya que arrojan evidencias del desempeño del estudiante, de su nivel de competencia y constituyen un insumo para proveer retroalimentación en la clase presencial. La actividad de preparación para la interacción en clase abarca 
desde los eventos cuatro al ocho con una propuesta de tareas para estimular la comunicación oral en clase a través de la negociación y el aspecto funcional de la lengua. Finalmente, la sesión presencial, cubre los
Modelo instruccional Blended-Flipped: personalización, flexibilización y metacognición para la nivelación en inglés en la educación superior María Catalina Caro Torres / Diana Angélica Parra Pérez / Alfredo José Averanga Murillo / Nelson Javier Corredor Plazas / Rosa Alejandra Medina Riveros

eventos seis al nueve, en los que se provocan los desempeños, el docente provee realimentación y tienen lugar la evaluación formativa y las oportunidades de interacción entre pares (ver figura 2).

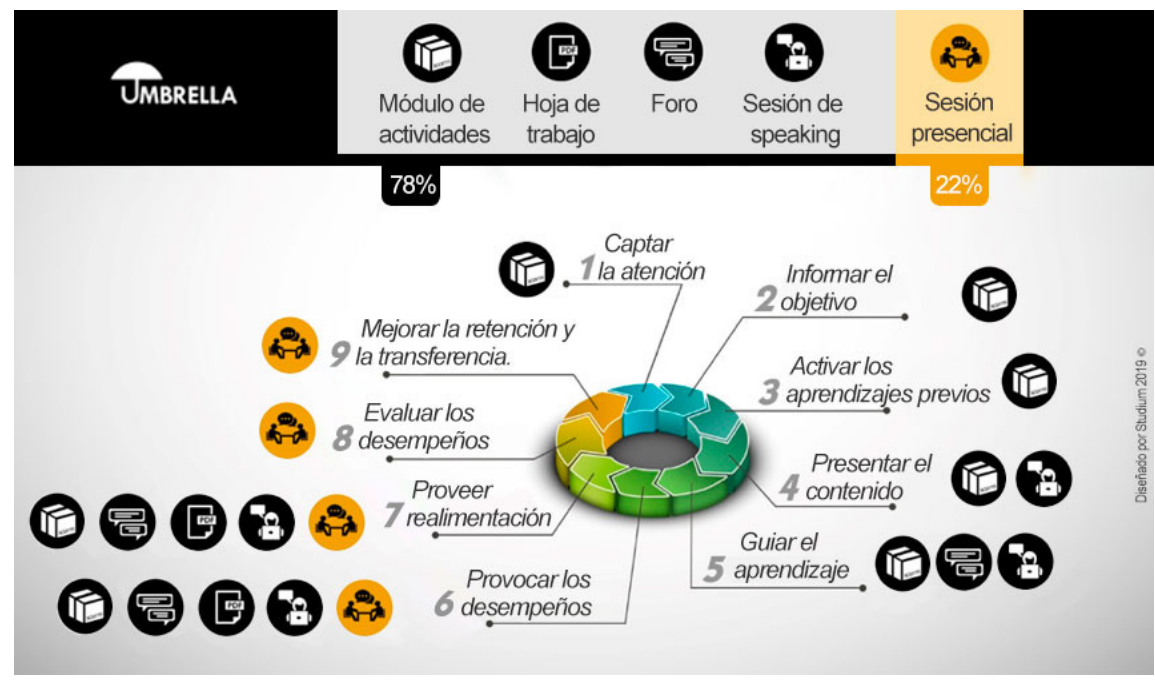

Figura 2. El modelo de enseñanza-aprendizaje híbrido-invertido.

Fuente: elaboración propia.

El equipo académico, conformado por una coordinadora y seis docentes del programa, desarrolló los contenidos pedagógicos y las actividades de aprendizaje. El equipo de desarrollo de recursos digitales, conformados por un coordinador, un diseñador y cuatro asistentes tecnológicos, desarrolló el estilo visual de los cursos y creó los objetos virtuales de aprendizaje con una herramienta de autor. Ambos equipos pilotearon los recursos y realizaron los ajustes necesarios hasta llegar a la versión final de los recursos de aprendizaje.

\section{Fase de implementación}

En esta fase los estudiantes reciben la plataforma de aprendizaje con todos los elementos de apoyo al aprendizaje (los contenidos, los recursos y los medios de interacción social, entre otros), adelantan el proceso de apropiación del conocimiento, desarrollan habilidades y demuestran comprensiones y desempeños. Los siete cursos del programa de inglés en modalidad híbrida-invertida se implementaron estimando cuatro horas de trabajo independiente de los estudiantes en la plataforma virtual y dos horas de asistencia a clase presencial semanal durante un periodo de 16 semanas por semestre. Teniendo en cuenta las necesidades de nivelación y personalización del aprendizaje del grupo objetivo, organizamos grupos de entre dos y seis estudiantes por docente. Realizamos la capacitación a los docentes en el manejo de LMS y programamos inducciones para los estudiantes para familiarizarlos con la metodología híbrida-invertida del programa y con el manejo de la plataforma de aprendizaje.

\section{Fase de evaluación}

La fase de evaluación es un proceso continuo y dinámico en el que se evalúa la calidad del programa de aprendizaje. La evaluación permitirá verificar si los objetivos de aprendizaje establecidos satisfacen las necesidades de aprendizaje de los estudiantes. Para llevar a cabo este proceso se determinan los criterios, los métodos y las herramientas de evaluación. Para evaluar el programa híbrido-invertido diseñamos encuestas de evaluación de la calidad de los recursos 
en el entorno virtual de aprendizaje y de la calidad de las prácticas de enseñanza en las clases presenciales. Las encuestas son respondidas por los docentes y por los estudiantes del programa al finalizar cada curso. Los resultados se analizan después de cada periodo académico y se crean planes de mejora. La evaluación de los aprendizajes incluye la evaluación formativa y la sumativa, generando así un proceso de evaluación continua que permite a los estudiantes verificar sus niveles de progreso en las competencias en inglés de cada nivel (Caro y Parra, 2018).

\section{Método de investigación}

Esta propuesta de investigación siguió un enfoque de corte cualitativo aplicando la teoría fundamentada, con la que se busca codificar los datos para desarrollar conceptos sobre un fenómeno particular (Glasser y Strauss, 2009). Basado en los procesos de codificación abierta, axial y selectiva se recolectaron los datos desde los registros documentales, las encuestas y las entrevistas. Los datos se analizaron mediante el software para investigación Atlas T.I., se siguió el procedimiento del método comparativo constante para determinar los códigos que en su abstracción generarán subcategorías y categorías conceptuales, según cada objetivo análisis.

El objetivo general de la investigación consistió en identificar, mediante las percepciones de los estudiantes, los alcances del modelo instruccional híbrido-invertido en la atención de las necesidades de nivelación en inglés de un grupo de estudiantes universitarios. El universo corresponde a un aproximado de 2240 estudiantes que cursan y han cursado inglés en el programa híbrido-invertido desde el 2007 hasta el 2019.

\section{Participantes}

Para este estudio se tomó una muestra representativa del $5 \%$ de dicha población, que corresponde a un aproximado de 112 participantes. Estos estudiantes son jóvenes entre los 17 y los 24 años, quienes están estudiando su carrera profesional en la institución de educación superior y que han realizado alguno de los 7 cursos de inglés, entre niveles A1.1 y B2.1 según el MCER (Consejo de Europa, 2001), ofrecidos por la institución mediante el programa híbrido-invertido.

\section{Instrumentos}

Para el tratamiento de los datos usamos las técnicas de recolección: análisis documental, encuesta y entrevista mediante los instrumentos: registros documentales, cuestionarios formales y entrevistas semiestructuradas.

\section{Registros documentales}

Utilizamos sistemáticamente dos registros documentales para la recolección de los datos referentes a la percepción de los estudiantes sobre el programa híbrido-invertido: 1) el formulario de inscripción en el que los estudiantes manifiestan las razones por las que quieren cursar inglés en esta modalidad, 2) los resultados de las encuestas de autoevaluación completadas por los estudiantes a mitad y final de cada curso. Estas evaluaciones son usadas en el programa para guiar a los estudiantes a identificar sus fortalezas y los aspectos que requieren mejorar, para crear un plan de acción oportuno.

\section{Cuestionarios formales}

La técnica de recolección de datos encuesta, mediante el instrumento cuestionario, se caracteriza por ser una prueba escrita compuesta por preguntas formulada a un grupo de personas con el fin de estudiar temas relacionados con el objeto de una investigación (Ibáñez, 2015). En esta investigación administramos al terminar cada periodo académico un cuestionario de satisfacción, diseñado para indagar sobre la percepción de los estudiantes respecto a su experiencia de aprendizaje, especialmente mediante las actividades virtuales: SCORM, worksheet (PDF), foro, verificador de aprendizaje, diario de aprendizaje y sesión presencial de aprendizaje.

\section{Entrevistas}

Las entrevistas semiestructuradas para la recolección de información incluyen al investigador como elemento fundamental del instrumento mismo, al ser él quien formula las preguntas y obtiene la información requerida. En este tipo de entrevistas 
se realizan preguntas específicas sobre las cuales el entrevistado tiene cierta libertad para responder y el entrevistador las recoge de forma literal (Abad, 1997). La entrevista semiestructurada nos permitió profundizar en la recolección de datos sobre la experiencia de aprendizaje de los estudiantes en el ambiente virtual y en el componente presencial del programa híbrido-invertido.

\section{Análisis y discusión de resultados}

Los resultados obtenidos mediante la triangulación y contraste de los datos recolectados, permitieron responder a la pregunta de investigación: ¿Qué percepción tienen los estudiantes sobre una propuesta de modelo instruccional para un ambiente híbrido de aprendizaje de inglés como lengua extranjera con un enfoque de aula invertida? Identificamos una categoría y dos subcategorías de análisis (Ver Tabla 1).

Tabla 1. Categorías y subcategorías de análisis

\begin{tabular}{|l|l|}
\multicolumn{1}{c|}{ Categoria } & \multicolumn{1}{c}{ Subcategorias } \\
\hline $\begin{array}{l}\text { Favorecimiento } \\
\text { del aprendizaje } \\
\text { de inglés }\end{array}$ & $\begin{array}{l}\text { 2. Incentiva el desarrollo de comporar barreras mediante } \\
\text { lamientos metacognitivos (Blakey y } \\
\text { tamida la personalización } \\
\text { Spence, 1990) y auto-regulados. }\end{array}$ \\
\hline
\end{tabular}

\section{Permite superar barreras mediante la flexibilidad y la personalización}

Los resultados del estudio informan que la flexibilidad y la personalización son los dos aspectos diferenciadores mediante los cuales los estudiantes caracterizan al modelo híbrido-invertido, denominado "Programa de Trabajo Independiente: Plan Umbrella”. Los participantes reconocen que estos dos elementos contribuyen a la superación de algunas de las barreras en su aprendizaje, como las barreras de falta de tiempo y las barreras afectivas.

\section{Flexibilidad vs. barreras de tiempo en el aprendizaje de inglés}

La flexibilidad puede ser categorizada en flexibilidad institucional y flexibilidad pedagógica (Barnett, 2014). La primera se relaciona con el interés ins- titucional en operacionalizar métodos altamente receptivos y personalizados basados en las necesidades de los aprendices (Butcher y Rose-Adams, 2015); la segunda, hace referencia al currículo, los roles del estudiante y el docente y la experiencia de aprendizaje-enseñanza. En lo concerniente a las barreras de tiempo, los estudiantes manifestaron que el programa híbrido-invertido favoreció su aprendizaje por la flexibilidad institucional, que les permitió lograr su meta de cursar el nivel de inglés, a pesar de sus limitaciones de tiempo: "es una oportunidad al alcance de las personas que no podemos cursar el nivel de otra manera debido a nuestras múltiples tareas" (Encuesta 14-1, 112). A lo anterior se suma la situación de los estudiantes de carreras como medicina, enfermería y fisioterapia, quienes deben cursar su práctica profesional en clínicas, hospitales o centros de salud, en horarios prolongados, impidiéndoles cursar el programa de inglés presencial compuesto por dos sesiones semanales en días y horarios diferentes: "Es un buen programa que facilita el aprendizaje del inglés a quienes por razones de tiempo no podemos asistir a varios horarios de clase a la semana" (Encuesta 14-1, 12).

Se ha afirmado que la tecnología en sí misma facilita el aprendizaje flexible (Papadima-Sophocleous y Loizides, 2016) y esto se corrobora con el modelo instruccional propuesto en este estudio. La estructura flexible de Plan Umbrella se evidencia en su diseño instruccional modular con enfoque pedagógico de aula invertida, que permite a los estudiantes realizar el $78 \%$ del curso de inglés en la plataforma virtual asincrónica, y el $22 \%$ en clases de dos horas semanales fijas, asignadas según el horario disponible de los participantes mismos. El programa posibilita el desarrollo de las actividades de aprendizaje virtuales en el tiempo de preferencia de los estudiantes y se acomoda al ritmo de estudio de cada individuo. Hallazgos similares se encuentran en un estudio con población universitaria en donde los estudiantes informaron altos niveles de satisfacción respecto a un sistema flexible de aprendizaje de lenguas, considerando que podían trabajar a su propio ritmo y que tenían la oportunidad de realizar los trabajos de forma autónoma (Chateau y Zumbihl, 2012). 
Desde la visión de la flexibilidad pedagógica en el modelo híbrido-invertido los estudiantes resaltaron la importancia del rol desempeñado por el docente para el logro de las metas de aprendizaje personales y del curso. En este programa, el docente es llamado tutor, ya que su rol primordial es el de acompañar a los estudiantes durante su proceso de aprendizaje y proveerles de experiencias de aprendizaje que respondan a sus necesidades: "[...] considero que el apoyo de la docente fue excelente, sus estrategias metodológicas favorecieron el aprendizaje" (Encuesta, 24). Lo anterior exige que el tutor planee las clases teniendo en cuenta los resultados de aprendizaje obtenidos por los estudiantes en las actividades virtuales: "el profesor observa cuales son los problemas que uno tiene y los ayuda a mejorar" (Encuesta 14-1, 31).

\section{Personalización vs. barreras afectivas en el aprendizaje de inglés}

En el aprendizaje de las lenguas extranjeras se ha demostrado la influencia de factores afectivos como la ansiedad, el autoconcepto, la autoestima, las actitudes y creencias, y los estilos de aprendizaje del estudiante (Mena, 2013). Los estudiantes de Plan Umbrella han enfrentado dificultades en procesos de aprendizaje de inglés previos, muy probablemente presentan niveles altos de intolerancia a la frustración, la desmotivación, el miedo a cometer errores o temores para expresar ideas en público haciendo uso de la segunda lengua: "Plan Umbrella es una excelente oportunidad para las personas que nos cuesta mucho el inglés ya que es un espacio más personalizado" (Encuesta 16-1, 33). Normalmente esta población se percibe a sí misma como incapaz para el aprendizaje de inglés e identifican con facilidad sus debilidades de aprendizaje antes que sus fortalezas. En palabras de Onwuegbuzie, Bailey y Daley "los estudiantes que tienen baja percepción sobre sus habilidades en la lengua extranjera tienden a tener bajos niveles de logro en la lengua extranjera" (2000, p. 12).

El análisis de las percepciones de los estudiantes informa que el programa favoreció su aprendizaje, motivación y auto-eficacia al contribuir en la superación de barreras afectivas: "me siento muy motivada en inglés" (Encuesta 18-1, 27); "pienso que fue una experiencia que me permitió perder el miedo a hablar" (Encuesta 14-1, 1). La auto-eficacia es entendida como la creencia de un individuo sobre sus propias capacidades para ejecutar acciones que le permitan lograr un desempeño esperado (Thai et al., 2017) y un ejemplo de cambio positivo en la auto-eficacia en inglés se evidencia en el siguiente extracto de entrevista: "logró quitar mi barrera frente al idioma y fortalecer cada una de las habilidades de los estudiantes" (Entrevista 16-2, 4). Se ha encontrado una correlación entre la autoeficacia con el desempeño de los estudiantes en ambientes híbridos de aprendizaje (Lynch y Dembo, 2004) y esto se corrobora en el presente estudio: "Estar en Plan Umbrella me ayudó a conocer mejor mis falencias y mis habilidades, en cuanto a mis notas tuve un mejoramiento notable" (Encuesta 17-1, 50).

Los mecanismos de seguimiento y apoyo al aprendizaje, los grupos de clase pequeños, las sesiones de tutoría personalizada enfocada al desarrollo de estrategias de aprendizaje y la realimentación individual clase a clase son elementos que nuestros estudiantes identifican como atributos del nivel de personalización de los procesos de enseñanza-aprendizaje del programa. Dicha personalización permitió a los estudiantes mejorar en aspectos como la auto-confianza, evidenciada en su entusiasmo, nivel de motivación, esfuerzo para estudiar y capacidad para afrontar las dificultades (Tunçel, 2015): "al ser grupos con un número más reducido de personas facilita el speaking y la resolución de inquietudes, crea también confianza en el estudiante" (Entrevista 18-2, 61) y el autoconcepto académico, es decir, la percepción de ellos mismos sobre sus intereses y desempeños (Zhan y Mei, 2013) en este caso sobre el aprendizaje de inglés: "Me siento satisfecho con el avance que he obtenido" (Encuesta 16-1, 56).

\section{Incentiva el desarrollo de comportamientos metacognitivos y autorreguladores}

Schunk (citado en Monge-López, Bonilla y AguilarFreyan, 2017) informa que "los estudiantes que 
presenten dificultades para manejar el tiempo y afrontar situaciones estresantes de la vida académica, y que tengan un pobre despliegue de estrategias de aprendizaje efectivas podrán tener mayor riesgo de presentar problemas académicos" (p. 63). Los resultados del presente estudio muestran que Plan Umbrella es un programa que favorece el aprendizaje de inglés al incentivar el desarrollo de comportamientos metacognitivos y autorreguladores en el aprendizaje. La personalización que maneja el programa, involucra retroalimentación y reflexión continua sobre el aprendizaje. Esto permite que los estudiantes estén atentos en su aprendizaje a indicadores que demuestren su progreso y sus necesidades de mejora, y que sean capaces de dar cuenta de cuánto han aprendido al finalizar cada corte académico: "siento que aprendí muchísimo y veo un avance en mí que antes no lo veía” (Encuesta 17-1, 52). Estas habilidades metacognitivas se ubican en la categoría más alta de aprendices metacognitivos propuesta por Perkins (1992), llamada aprendices reflexivos, que se caracterizan por su capacidad de manejar estratégicamente su pensamiento y de reflexionar sobre su aprendizaje, considerando lo que le es útil o efectivo y lo que no lo es: “[...] este semestre aprendí mucho ...tuve un mejor desempeño a diferencia de los niveles presenciales, mi nivel de speaking mejoró muchísimo a comparación de los demás niveles y mejoré mi competencia en las demás actividades" (Encuesta 14-2, 45).

La conciencia que generan los estudiantes sobre su propio aprendizaje se debe también al desarrollo de estrategias de autorregulación, que tienen lugar cuando hay un monitoreo y una adaptación intencional de las prácticas académicas para afrontar satisfactoriamente los retos de aprendizaje (Vermunt, 2005). Al iniciar cada semestre académico, los estudiantes son guiados en el uso de estrategias de manejo y de organización del tiempo, lo cual contribuye a su proceso de adaptación al ambiente virtual de aprendizaje, e incentiva el logro de las metas de estudio y la preparación de actividades virtuales semanales, antes de asistir a la clase.

Una de las dimensiones de evaluación de estrategias de autorregulación propuesta por Vermunt y
Rijswijk (1988) es la autorregulación de los procesos, los resultados y los contenidos. Esta estrategia se refiere a los comportamientos metacognitivos propios, diferentes a los fijados por el docente, como la evaluación de progreso de su propio aprendizaje: "fue un plan que me ayudó a fortalecer mi proceso de aprendizaje del inglés" (Encuesta 18-1,21). Los resultados aquí expuestos nos permitieron determinar que el programa de inglés híbrido-invertido incentiva al desarrollo de comportamientos metacognitivos y de autorregulación.

\section{Conclusiones}

La creación de un modelo instruccional híbrido-invertido significó institucionalmente la posibilidad de ofrecer a los estudiantes una alternativa flexible y personalizada para cursar su asignatura de inglés. Este estudio se enfocó en el análisis de la percepción de los estudiantes sobre el modelo instruccional híbrido-invertido, diseñado en respuesta a las necesidades de aprendizaje de los estudiantes mismos (Kakosimos, 2015). Los resultados demuestran que el modelo instruccional híbrido-invertido favorece el aprendizaje de inglés, ya que permite a los estudiantes superar barreras de tiempo y barreras afectivas gracias a su nivel de flexibilización y personalización; también porque promueve el desarrollo de comportamientos metacognitivos y autorreguladores en los estudiantes que lo cursan, lo cual concuerda con hallazgos encontrados en otros estudios (Gilboy, Heinerichs y Pazzaglia, 2015; Sun, Xie y Anderman, 2018; Yang, Yin y Wang, 2018).

Este estudio muestra una vía mediante la cual la tecnología puede ser positivamente integrada a los procesos de enseñanza-aprendizaje de inglés como lengua extranjera, puesto que permite un enfoque en el que el estudiante es el centro de la educación y exalta la tecnología como el medio facilitador en el proceso de flexibilización. En el marco de este enfoque también se señala una forma de adaptar las prácticas de enseñanza a las necesidades específicas de los estudiantes de la actualidad y contribuir a su éxito académico en la educación superior. 


\section{Referencias}

Abad, M. F. (1997). Investigación evaluativa en documentación: Aplicación a la documentación médica. Universitat de València. https://dialnet.unirioja.es/ servlet/libro? codigo $=17793$

Ammar Abdullah, M. I. (2017). The effects of enhancing prospective EFL teachers' knowledge management strategies in virtual learning environments on their ideational flexibility and engagement. International Journal of Applied Linguistics and English Literature, 6(2), 154-172. http://dx.doi.org/10.7575/aiac. ijalel.v.6n.2p.154

Alonso, F., López, G., Manrique, D. y Viñes, J. (2005). An instructional model for web-based elearning education with a blended learning process approach. British Journal of Education Technology, 36(2), 217-235. https://doi.org/10.1111/j.1467-8535.2005.00454.x

Avello, R. y Duart, J. M. (2016). Nuevas tendencias de aprendizaje colaborativo en e-learning: claves para su implementación efectiva. Estudios Pedagógicos, 42(1), 271-282. http://dx.doi.org/10.4067/ S0718-07052016000100017

Barnett, R. (2014). Conditions of flexibility: Securing a more responsive higher education system. Higher Education Academy, 5. https://www.heacademy. ac.uk/sites/default/files/resources/FP_conditions_ of_flexibility.pdf

Blakey, E. y Spence, S. (1990). Developing metacognition. ERIC Information Center Resources. https:// eric.ed.gov/?id=ED327218

Blau, I. y Shamir-Inbal, T. (2017). Re-designed flipped learning model in an academic course: The role of co-creation and co-regulation. Computers \& Education, 115, 69-81. https://doi.org/10.1016/j. compedu.2017.07.014

Broadbent, J. (2017). Comparing online and blended learner's self-regulated learning strategies and academic performance. The Internet and Higher Education, 33, 24-32. https://psycnet.apa.org/ doi/10.1016/j.iheduc.2017.01.004

Butcher, J. y Rose-Adams, J. (2015). Part-time learners in open and distance learning: revisiting the critical importance of choice, flexibility and employability. Open Learning, 30(2), 127-137. https://doi.org/10.1 080/02680513.2015.1055719

Caro, C. y Parra, D. (2018). Primero yo, segundo yo y lo que quede para mi aprendizaje: autoevaluación como un trabajo de egoísmo positivo. En M. A. Santos,
Evaluar para aprender: Investigación-acción en la Universidad de La Sabana, (pp. 189-203). https:// www.unisabana.edu.co/feria-del-libro-2018/evaluar-para-aprender-investigacion-accion-en-la-universidad-de-la-sabana/

Chateau, A. y Zumbihl, H. (2012). Learners' Perceptions of the Pedagogical Relations in a Flexible Language Learning System. Computer Assisted Language Learning, 25(2), 165-179. https://doi.org/10.1080/09588221.2 011.636053

Chilingaryan, K. y Zvereva, E. (2017). Methodology of flipped classroom as a learning technology in foreign language teaching. Procedia-Social and Behavioral Sciences, 237, 1500-1504. https://doi.org/10.1016/j. sbspro.2017.02.236

Clark, D. (2014). Instructional System Design: The ADDIE Model. http://www.nwlink.com/ donclark/ index.html

Consejo de Europa (2001). Marco común europeo de referencia para las lenguas: aprendizaje, enseñanza, evaluación. Anaya. https://cvc.cervantes.es/ ensenanza/biblioteca_ele/marco/

El-Banna, W., Whitlow, M. y McNelis, A.M. (2017). Flipping around the classroom: Accelerated Bachelor of Science in Nursing students' satisfaction and achievement. Nurse Education Today, 56, 41-46. https://doi.org/10.1016/j.nedt.2017.06.003

Evseeva, A. y Solozhenko, A. (2015). Use of Flipped Classroom Technology in Language Learning. Procedia - Social and Behavioral Sciences, 206, 205-209. https://doi.org/10.1016/j.sbspro.2015.10.006

Gagné, R. M. y Dick, W. (1983). Instructional Psychology. Annual Review of Psychology, 34, 261-295. https:// doi.org/10.1146/annurev.ps.34.020183.001401

García-Sánchez, S. y Santos-Espino, J. M. (2017). Empowering pre-service teachers to produce ubiquitous flipped classes. Profile Issues in Teachers ' Professional Development, 19(1) 169-185. http:// dx.doi.org/10.15446/profile.v19n1.53857

Gilboy, M., Heinerichs, S. y Pazzaglia, G. (2015). Enhancing Student Engagement Using the Flipped Classroom. Journal of Nutrition Education and Behavior, 47(1), 109-114. https://doi.org/10.1016/j. jneb.2014.08.008

Glasser, B. G. y Strauss, A. L. (2009). The discovery of grounded theory: Strategies for qualitative research. Transaction Publishers. http://www.sxf.uevora.pt/ wp-content/uploads/2013/03/Glaser_1967.pdf 
Hao, Y. y Lee, K. (2016). Teaching in flipped classrooms: Exploring pre-service teachers' concerns. Computers in Human Behavior, 57, 250-260. https://doi. org/10.1016/j.chb.2015.12.022

Hirata, Y. y Hirata, Y. (2008) Japanese Students' Attitudes towards Hybrid Learning. En Fong J., Kwan R., Wang F.L. (eds) Hybrid Learning and Education. ICHL 2008. Lecture Notes in Computer Science, Berlin, Heidelberg. https://link.springer.com/ chapter/10.1007/978-3-540-85170-7_39

Hubackova, S. y Semradova, I. (2016). Evaluation of blended learning. Procedia - Social and Behavioral Sciences, 217, 551-557. https://doi.org/10.1016/j. sbspro.2016.02.044

Ibáñez, J. (2015) Métodos, técnicas e instrumentos de la investigación criminológica. Dykinson.

Janthon, U., Songkran, N. y Koraneekij, P. (2015). Workbased blended learning and technological scaffolding system to enhance communication skills for caregivers under local administrative organization, ministry of interior, Thailand (part I). Procedia Social and Behavioral Sciences, 174, 984-991. https:// doi.org/10.1016/j.sbspro.2015.01.722

Jeong, K. O. (2017). The use of Moodle to enrich flipped learning for English as a foreign language education. Journal of Theoretical y Applied Information Technology, 95(18), 4845-4852. http://www.jatit.org/ volumes/Vol95No18/23Vol95No18.pdf

Kakosimos, K. E. (2015). Example of a micro-adaptive instruction methodology for the improvement of flipped-classrooms and adaptive-learning based on advanced blended-learning tools. Education for chemical engineers, 12, 1-11. https://doi.org/10.1016/j. ece.2015.06.001

Keong, Y. C. y Subhi, S. N. (2015). The role of knowledge sharing in the learning process among Iraqi $\mathrm{EFL}$ postgraduate students in UKM. International Journal of Education and Research, 3(12), 185-198. https:// doi.org/10.1080/14703297.2015.1122544

Khlaisang, J. Y. y Likhitdamrongkiat, M. (2015). E-learning system in blended learning environment to enhance cognitive skills for learners in higher education. Procedia - Social and Behavioral Sciences, 174, 759-767. https://doi.org/10.1016/j.sbspro.2015.01.612

Klimova, B. y Kacetl, J. (2015). Hybrid learning and its current role in the teaching of foreign languages. Procedia - Social and Behavioral Sciences, 182, 477481. https://doi.org/10.1016/j.sbspro.2015.04.830
Modelo instruccional Blended-Flipped: personalización, flexibilización y metacognición para la nivelación en inglés en la educación superior María Catalina Caro Torres / Diana Angélica Parra Pérez / Alfredo José Averanga Murillo / Nelson Javier Corredor Plazas / Rosa Alejandra Medina Riveros

Kofar, G. (2016). A Study of EFL instructors' perceptions of blended learning. Procedia - Social and Behavioral Sciences, 232, 736-744. https://doi.org/10.1016/j. sbspro.2016.10.100

Krasnova, T. y Demeshko, M. (2015). Tutor-mediated Support in Blended Learning. Procedia - Social and Behavioral Sciences, 166, 404-408. https://doi. org/10.1016/j.sbspro.2014.12.544

Liu, D. (2016). The Reform and Innovation of English Course: A Coherent Whole of Mooc, Flipped Classroom and EsP. Procedia-Social and Behavioral Sciences, 232, 280-286. https://doi.org/10.1016/j. sbspro.2016.10.021

Loya, A., Gopal, A., Shukla, I., Jermann, P. y Tormey, R. (2015). Conscientious behaviour, flexibility and learning in massive open on-line courses. Procedia Social and Behavioral Sciences, 191, 519-525. https:// doi.org/10.1016/j.sbspro.2015.04.686

Lynch, R. y Dembo, M. (2004). The relationship between self-regulation and online learning in a blended learning context. International Review of Research in Open and Distance Learning, 5(2), 1-16. https:// doi.org/10.19173/irrodl.v5i2.189

Matukhin, D. y Zhitkova, E. (2015). Implementing blended learning technology in higher professional education. Procedia - Social and Behavioral Sciences, 206, 183-188. https://doi.org/10.1016/j. sbspro.2015.10.051

Mena, T. (2013). Factores afectivos que inciden en el aprendizaje de una lengua extranjera: motivación. [Tesis de maestría, Universidad de Oviedo]. Repositorio institucional Uniovi. http://digibuo.uniovi.es/dspace/ bitstream/10651/18314/6/TFM_\%20MenaBenet.pdf

Onwuegbuzie, A., Bailey, P. y Daley, C. (2000). Cognitive, Affective, Personality, and Demographic Predictors of Foreign-Language Achievement, The Journal of Educational Research, 94(1), 3-15. https://doi. org/10.1080/00220670009598738

Osorio, L. A. (2010). Características de los ambientes híbridos de aprendizaje: estudio de caso de un programa de posgrado de la Universidad de los Andes. Revista de Universidad y Sociedad del Conocimiento, 7(1), 01-09. http://www.redalyc.org/pdf/780/78012953004.pdf

Papadima-Sophocleous, S. y Loizides, F. (2016, agosto). Exploring the benefits and disadvantages of introducing synchronous to asynchronous online technologies to facilitate flexibility in learning. En S. Papadima-Sophocleous, L. Bradley y S. Thouësny 
(Eds), CALL communities and culture - short papers from EUROCALL 2016 (pp. 363-368). https://doi. org/10.14705/rpnet.2016.eurocall2016.589

Parra, D. y Medina, R. (2014, 17-19 de noviembre). Unleashing the power of blended learning and flipped classroom for English as a foreign language learning: Three spheres of challenges and strategies in a higher education institution in Colombia. Séptima Conferencia Internacional de Educación, Investigación en Innovación, ICERI, Sevilla, España. https://doi. org/10.13140/rg.2.1.2559.2725

Pavla, S., Hana, V. y Jan, V. (2015). Blended Learning: Promising strategic alternative in higher education. Procedia - Social and Behavioral Sciences, 171, 12451254. https://doi.org/10.1016/j.sbspro.2015.01.238

Perkins, D. (1992). Smart Schools: Better Thinking and Learning for Every Child. Free Press.

Porter, W. y Graham, C. (2016). Institutional drivers and barriers to faculty adoption of blended learning in higher education. British Journal of Educational Technology, 47(4), 748-762. https://doi.org/10.1111/ bjet.12269

Regalón, L. y Leyva, J. (2014). Impacto de la enseñanza-aprendizaje del inglés con "At your Pace" en las carreras de Derecho y Estudios Socioculturales. Pedagogía Universitaria, 19(1), 89-101. https://go.gale.com/ps/ anonymous?id=GALE $\% 7 C A 466940253 \&$ sid $=-$ googleScholar\&v=2.1\&it=r\&linkaccess=abs\&issn= $16094808 \& \mathrm{p}=\mathrm{AONE} \& \mathrm{sw}=\mathrm{w}$

Monge-López, D., Bonilla, R. y Aguilar-Freyan, W. (2017). El inventario de estrategias de autorregulación: traducción al español, características psicométricas preliminares y su relación con variables sociodemográficas en una muestra de estudiantes universitarios. Avances en Psicología Latinoamericana, 35(1), 61-78. https://doi.org/10.12804/revistas.urosario. edu.co/apl/a.3729

Sergis, S., Sampson, D. G. y Pelliccione, L. (2018). Investigating the impact of Flipped Classroom on students' learning experiences: A self-determination theory approach. Computers in Human Behavior, 78, 368-378. https://doi.org/10.1016/j. chb.2017.08.011

Sohrabi, B. y Iraj, H. (2016). Implementing flipped classroom using digital media: A comparison of two demographically different groups perceptions. Computers in Human Behavior, 60, 514-524. https:// doi.org/10.1016/j.chb.2016.02.056
Sriarunrasmee, J., Techataweewan, W. y Mebusaya, R. (2015). Blended Learning Supporting SelfDirected Learning and Communication Skills of Srinakharinwirot University's First Year Students. Procedia - Social and Behavioral Sciences, 197, 15641569. https://doi.org/10.1016/j.sbspro.2015.07.111

Sun, Z., Xie, K. y Anderman, L. H. (2018). The role of self-regulated learning in students' success in flipped undergraduate math courses. The Internet and Higher Education, 36, 41-53. https://psycnet.apa. org/doi/10.1016/j.iheduc.2017.09.003

Thai, N., De Wever, B. y Valcke, M. (2017). The impact of a flipped classroom design on learning performance in higher education: Looking for the best "blend" of lectures and guiding questions with feedback. Computers y Education, 107, 113-126. https://doi. org/10.1016/j.compedu.2017.01.003

Tunçel, H. (2015). The relationship between self-confidence and learning Turkish as a foreign language. Educational Research and Reviews, 10(18), 25752589. https://eric.ed.gov/?id=EJ1078234

Vermunt, J. D. (2005). Relations between student learning patterns and personal and contextual factors and academic performance. Higher Education, 49(3), 205-234. https://doi.org/10.1007/s10734-0046664-2

Vermunt, J. D. y Rijswijk, V. (1988). Analysis and development of students' skills in selfregulated learning. Higher Education, 17(6), 647-682. http://www.jstor. org/stable/3447008

Vidal, M., Rivera, N., Nolla, N., Morales, I. y Vialart, M. (2016). Aula invertida, nueva estrategia didáctica. Revista Cubana de Educación Médica Superior, 30(3), 678-688. http://ems.sld.cu/index.php/ems/article/ view/855/432

Wang, F. (2017). An exploration of online behaviour engagement and achievement in flipped classroom supported by learning management system. Computers \& Education, 114, 79-91. https://doi. org/10.1016/j.compedu.2017.06.012

Wright, B. M. (2017). Blended learnings student perception of face-to-face and online EFL lessons. Indonesian Journal of Applied Linguistics, 7(1), 64-71. https://doi.org/10.17509/ijal.v7i1.6859

Yalçınkaya, D. (2015). Why is Blended Learning for Vocationally Oriented Language Teaching? Procedia - Social and Behavioral Sciences, 174, 1061-1068. https://doi.org/10.1016/j.sbspro.2015.01.795 
Yang, J., Yin, C. X. y Wang, W. (2018). Flipping the classroom in teaching Chinese as a foreign language. Language Learning y Technology, 22(1), 16-26. https://dx.doi.org/10125/44575

Zhan, Z. y Mei, H. (2013). Academic self-concept and social presence in face-to-face and online learning: Perceptions and effects on students' learning achievement and satisfaction across environments. Computers and Education. 69, 131-138. https://doi. org/10.1016/j.compedu.2013.07.002

Zhu, Y., Au, W. y Yates, G. (2016). University students' self-control and self-regulated learning in a blended course. The Internet and Higher Education, 30, 54-62. https://doi.org/10.1016/j.iheduc.2016.04.001 
